\title{
Detailed radiological study of the patent ductus arteriosus: a computed tomography study in the Polish population
}

\author{
M. Krupiński', M. Irzyk¹, Z. Moczulski', R. Banyś', M. Kuniewicz², M. Urbańczyk-Zawadzka \\ 'Department of Radiology and Diagnostic Imaging, John Paul II Hospital, Krakow, Poland \\ ${ }^{2}$ Department of Anatomy, Jagiellonian University Medical College, Krakow, Poland
}

[Received: 5 May 2019; Accepted: 4 October 2019]

Background: The aim of the study was to perform qualitative and quantitative computed tomography (CT) angiography-based evaluation of patent ductus arteriosus (PDA) morphology and its influence on morphology of the great vessels. Materials and methods: Two-thousand twenty-two patients underwent 64-slice or dual-source CT and were retrospectively screened for the presence of PDA. Those who had presence of PDA underwent evaluation of its anatomy and morphology.

Results: Thirty-two adult patients with PDA were evaluated (mean age $41.4 \pm$ \pm 17.4 years). Subjects with PDA had a higher value of aortic isthmus $(p=0.0148)$, main pulmonary artery $(p<0.0001)$, right $(p=0.0007)$ and left $(p=0.0074)$ pulmonary arteries diameters than individuals from control group ( 16 adults, median age $43.3 \pm 12.4$ years). Types $A, B, C, D$, and $E$ of PDA morphology occurred in $16(50 \%), 3(9 \%), 9(28 \%), 2(6 \%)$, and $2(6 \%)$ patients, respectively. Subjects with the type $A$ configuration of PDA tended to have a larger diameter at the aortic orifice (10.2 $\pm 5.2 \mathrm{~mm}$ vs. $6.4 \pm 4.9 \mathrm{~mm}, p=0.09)$ and a larger maximal diameter $(10.3 \pm 5.3 \mathrm{~mm}$ vs. $7.1 \pm 4.7 \mathrm{~mm}, p=0.14)$ compared to subjects with the type $C$ configuration. The values of minimal, mean, and maximal diameters of PDA were $4.7 \pm 1.9 \mathrm{~mm}, 7.0 \pm 3.2 \mathrm{~mm}$, and $9.4 \pm 5.0 \mathrm{~mm}$, respectively. The Spearman correlation coefficient between the main pulmonary artery and PDA diameters demonstrated a good correlation for minimal $(r=0.70, p<0.001)$, mean $(r=0.62, p=001)$, and maximal $(r=0.60, p=0.0003)$ PDA diameters. Conclusions: Computed tomography enables quantitative and qualitative evaluation of PDA, including its type of morphology, length, and diameters. In the evaluated adult population with PDA, the majority of patients had dilation of the aortic isthmus and pulmonary arteries. PDA diameters correlate with diameters of the pulmonary arteries and this correlation is strongest between PDA diameter at the narrowest site and main pulmonary artery. (Folia Morphol 2020; 79, 3: 462-468)

Key words: multi-slice computed tomography, patent ductus arteriosus, cardiovascular abnormalities 


\section{INTRODUCTION}

In foetal life, the arterial duct typically connects the descending thoracic aorta and the proximal left pulmonary artery; however, some variations in its origin and insertion may occur [1]. After birth, the arterial duct typically closes functionally within 24-48 hours and anatomically within 1 month [10]. Persistence of the arterial duct for more than 3 months after birth is a congenital heart defect known as patent ductus arteriosus (PDA), which occurs in 1 out of 2000 full-term born children and comprises $5-10 \%$ of all congenital heart diseases [3, 12]. Its clinical presentation may vary from completely asymptomatic to symptoms of congestive heart failure and is related to the magnitude of the shunt [14]. The value of the shunt depends on the length, diameter, and morphology of the PDA [10] and is also known to have an impact on morphology of the heart and great vessels [14]. The shunt is calculated as the ratio between flow through the pulmonary artery and the aorta $[2,16]$. According to the value of this ratio, the shunt may be classified as small (ratio $<1.5$ ), moderate (ratio 1.5-2.2) or large (ratio > 2.2) [16]. Diagnostic imaging evaluation of the PDA may be performed either noninvasively by echocardiography, computed tomography (CT), and magnetic resonance or invasively by cardiac catheterisation [10, 14]. CT, unlike other methods, does not allow for flow quantification; however, it does enable a detailed evaluation of PDA morphology and size together with potential complications such as aneurysms, thrombi, and calcifications $[1,10,14]$. Such detailed evaluation is useful in the diagnosis of PDA and is crucial for the planning of PDA percutaneous closure [9]. Moreover, $\mathrm{CT}$ enables quantitative evaluation of great vessel morphometry including potential pulmonary artery hypertension and Eisenmenger syndrome [5]. To date, an angiographically based morphometric classification of the PDA by Krichenko et al. [11] has been utilized (Fig. 1). However, it is limited since it is a 2-dimensional image and has lower diagnostic accuracy in evaluation of thrombi and calcifications when compared to $C T$ [11]. Magnetic resonance enables quantification of the shunt through the PDA, but its diagnostic value in PDA morphology evaluation is limited [2]. Qualitative and quantitative analysis of PDA morphometric details using CT is a new perspective which is lacking in previously published literature. Previous CT-based analyses $[8,10]$ concentrated mostly on the detection and incidence of PDA

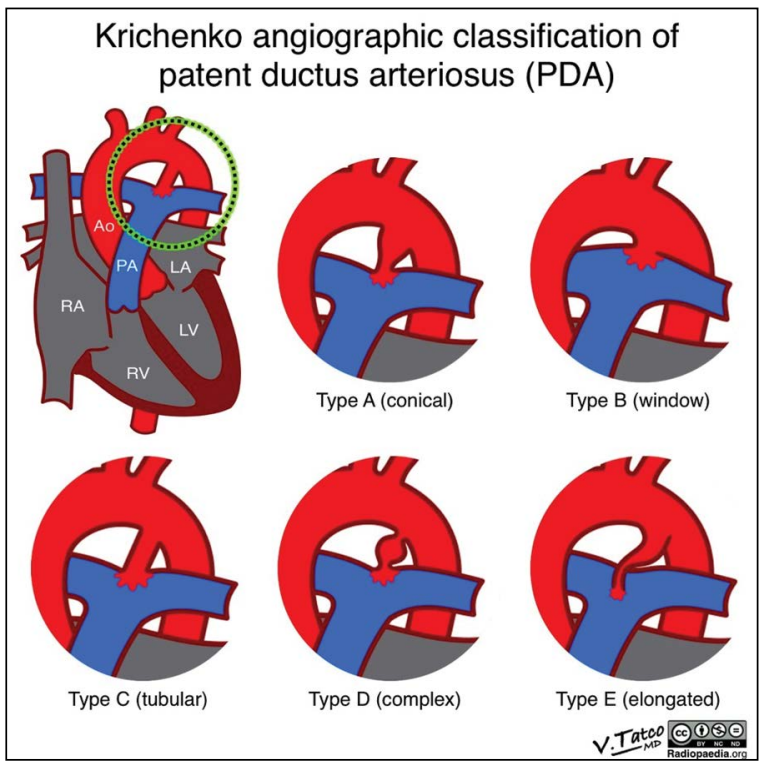

Figure 1. Variations in patent ductus arteriosus (PDA) configuration illustrated with the classification of Krichenko et al. [11]: A. Type A: conical ductus with prominent aortic ampulla and constriction near the pulmonary artery end; B. Type B: window-like (wide and very short) ductus; C. Type C: tubular ductus without constrictions; D. Type D: complex ductus with multiple constrictions; E. Type E: elongated ductus with constriction remote from the anterior edge of the trachea (when viewed on lateral angiography). Source: https://radiopaedia.org/cases/krichenko-angiographic-classification-of-patent-ductus-arteriosus-illustration. CC licence, BY, NC, ND.

in the population. Therefore, the aim of our study was to perform a CT angiography-based evaluation of PDA morphology and its influence on great vessel morphology.

\section{MATERIALS AND METHODS}

Two-thousand twenty-two patients underwent $\mathrm{CT}$ angiography of the thoracic aorta and were retrospectively screened for the presence of PDA. CT examinations were performed during a period of 10 consecutive years using 64-slice (Somatom Sensation 64 Cardiac, Siemens, Erlangen, Germany) or dual-source CT (Somatom Definition, Siemens, Erlangen, Germany). Patients underwent CT angiography due to known or suspected aortic disease including aortic aneurysm and congenital heart disease. Exclusion criteria for performing $\mathrm{CT}$ angiography were: pregnancy, known severe allergy to iodinated contrast, impaired renal function (serum creatinine $\geq 1.5 \mathrm{mg} / \mathrm{dL}$ ), and thyroid disorders [13]. Contrast-enhanced acquisitions were performed during inspiratory breath hold with a collimation of $0.6 \mathrm{~mm}$. Field of view extended from the level above the aortic arch to the level below the diaphragm for the thoracic aorta. The bolus 


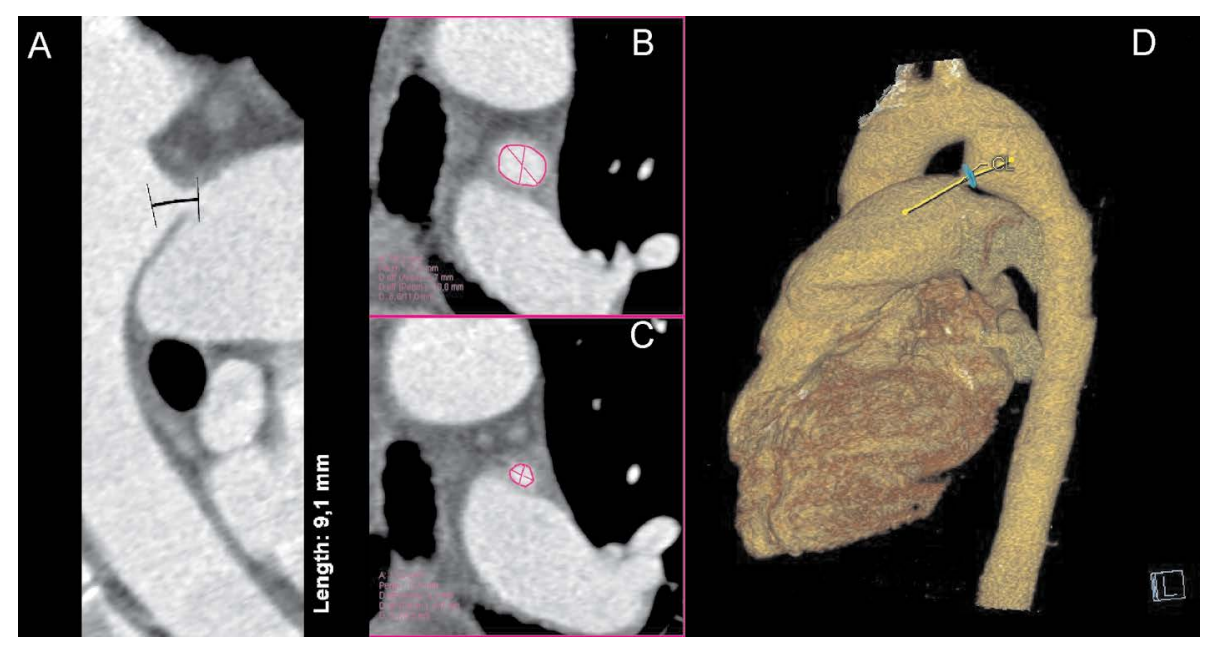

Figure 2. Multiplanar (A, B, C) and volume rendered technique reconstructions (D) of patent ductus arteriosus. Quantitative evaluation of length (A), aortic and pulmonary orifices $(\mathbf{B}, \mathbf{C})$.

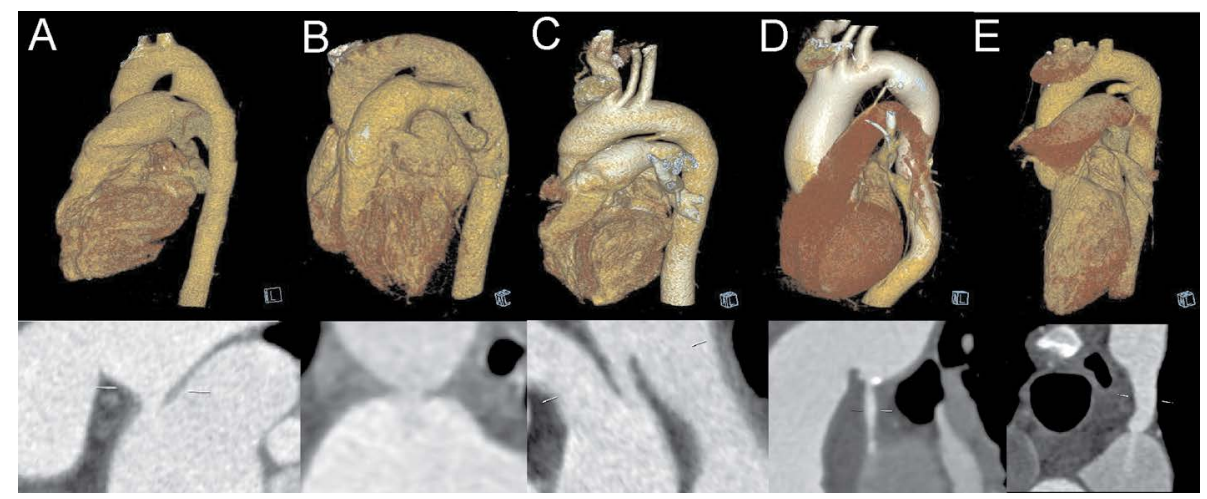

Figure 3. Multiplanar and volume rendered technique reconstructions of patent ductus arteriosus morphology types: A. "Conical” with wide aortic and narrower pulmonary orifices; B. "Window" with very short length, wide aortic and pulmonary orifices; C. "Tubular" with wide aortic and pulmonary orifices; D. “Complex" with multiple constrictions; E. "Elongated” with distal constriction.

tracking method was used with the region of interest in the aortic arch and the scan was initiated when the density reached 180 Hounsfield Units. An iodinated contrast agent was injected at a rate of $5 \mathrm{~mL} / \mathrm{s}$ [13]. Images were reconstructed with an image matrix of $512 \times 512$ pixels. Post-processing and study evaluation were performed using a dedicated workstation (Syngovia, Siemens, Erlangen, Germany). Patients were assessed for the presence of PDA using axial, curved multiplanar and volume-rendered technique reconstructions. In subjects with PDA, CT evaluation included quantitative and qualitative analysis of the aorta, PDA, and pulmonary arteries. Quantitative analysis included measurement of aortic, PDA, main pulmonary, and right and left pulmonary artery diameter (defined as the mean value of two perpendicular diameters) at the level of the ascending aorta at its maximal diameter, site of PDA, descending aorta at its maximal diameter, main pulmonary artery (MPA) at its maximal diameter, and right and left pulmonary arteries at their maximal diameters. Evaluation of PDAs included measurement of their length, diameter at their aortic and pulmonary endings (Fig. 2) and in the middle part. Calculation of their minimal, maximal, and mean diameter was also performed. Qualitative analysis included evaluation for the presence of PDA thrombus, calcification $[1,10,14]$, PDA morphometry (Fig. 3) [11], aortic dilatation (defined as $\geq 37 \mathrm{~mm}$ in the ascending, $\geq 30 \mathrm{~mm}$ in the isthmus and descending aorta) and dilatation of the pulmonary arteries (defined as $\geq 29 \mathrm{~mm}$ in the MPA, $\geq 20 \mathrm{~mm}$ in the left and right pulmonary arteries) [13]. 
Table 1. The results of quantitative and qualitative computed tomography analysis of aorta and pulmonary arteries in patients with patent ductus arteriosus (PDA) compared with control group

\begin{tabular}{lccc}
\hline Variable & $\begin{array}{c}\text { Patients with PDA ( } \mathbf{n}=\mathbf{3 2}) \\
\text { Mean } \pm \text { SD }\end{array}$ & $\begin{array}{c}\text { Control group ( } \mathbf{n}=\mathbf{1 6}) \\
\text { Mean } \pm \text { SD }\end{array}$ & P \\
\hline Ascending aorta diameter [mm] & $33.4 \pm 7.3$ & $32.4 \pm 4.6$ & 0.6554 \\
Ascending aorta dilatation & 9 & 3 & 0.72522 \\
Aortic isthmus diameter [mm] & $28.8 \pm 6.3$ & $24.5 \pm 3.5$ & 0.0148 \\
Aortic isthmus dilatation & 17 & 3 & 0.031240 \\
Descending aorta diameter [mm] & $24.6 \pm 2.8$ & $22.7 \pm 3.8$ & 0.0833 \\
Descending aorta dilatation & 1 & 1 & 1 \\
MPA diameter [mm] & $34.0 \pm 6.5$ & $21.7 \pm 3.7$ & $<0.0001$ \\
MPA dilatation & 27 & 1 & $<0.0001$ \\
RPA diameter [mm] & $24.3 \pm 5.4$ & $19.2 \pm 2.1$ & 0.0007 \\
RPA dilatation & 26 & 6 & 0.007660 \\
LPA diameter [mm] & $22.4 \pm 3.9$ & $19.6 \pm 1.1$ & 0.0074 \\
LPA dilatation & 26 & 8 & 0.042101 \\
\hline
\end{tabular}

LPA — left pulmonary artery; MPA — main pulmonary artery; RPA — right pulmonary artery

Table 2. The results of quantitative and qualitative computed tomography analysis of patent ductus arteriosus (PDA)

\begin{tabular}{lccccc}
\hline Type of PDA morphology & Type A $(\mathbf{n}=\mathbf{1 6})$ & Type B ( $\mathbf{n}=\mathbf{3})$ & Type C ( $\mathbf{n}=\mathbf{9})$ & Type D ( $\mathbf{n}=\mathbf{2})$ & Type E (n= 2) \\
\hline Minimal diameter [mm] & $4.8 \pm 2.2$ & $5.8 \pm 2.3$ & $4.3 \pm 2.0$ & $3.8 \pm 0.4$ & $4.7 \pm 0.4$ \\
Maximal diameter [mm] & $10.3 \pm 5.3$ & $13 \pm 4.2$ & $7.1 \pm 4.7$ & $9.0 \pm 5.7$ & $7.8 \pm 1.4$ \\
Mean diameter [mm] & $7.4 \pm 3.0$ & $9.8 \pm 3.8$ & $5.8 \pm 3.5$ & $5.8 \pm 2.6$ & $5.8 \pm 0.2$ \\
Aortic orifice diameter [mm] & $10.2 \pm 5.2$ & $12.2 \pm 10.2$ & $6.4 \pm 4.9$ & $5.2 \pm 5.8$ & $4.5 \pm 0.4$ \\
Pulmonary orifice diameter [mm] & $5.1 \pm 2.7$ & $8.7 \pm 7.8$ & $5.5 \pm 4.2$ & $3.8 \pm 0.4$ & $3.3 \pm 4.0$ \\
PDA length [mm] & $7.5 \pm 3.0$ & $3.0 \pm 1$ & $5.4 \pm 1.5$ & $17 \pm 4.2$ & $14.5 \pm 0.7$ \\
\hline
\end{tabular}

\section{Statistical analysis}

Variables were presented as mean \pm standard deviation and compared to a randomly chosen control group using the Student's t-test. Spearman correlation coefficient analyses were performed for comparison of measurements between PDA and pulmonary arteries. In qualitative analysis, the $\chi^{2}$ test with Yates modification was used. $\mathrm{P}<0.05$ was considered as statistically significant. The statistical analysis was performed using Statistica 10 software (StatSoft Inc.). The local bioethics committee approved the protocol and classified it as a retrospective study.

\section{RESULTS}

Thirty-two adult patients with PDA were evaluated (mean age $41.4 \pm 17.4$ years). Subjects with PDA had larger diameter of aortic isthmus $(p=0.0148)$, MPA ( $p<0.0001)$, and right $(p=0.0007)$ and left ( $p=0.0074)$ pulmonary arteries than individuals from the control group (16 adults, median age $43.3 \pm$ \pm 12.4 years). Sixteen (half of the evaluated population) randomly chosen patients were also evaluated as a control group. The detailed qualitative and quantitative analysis of aortic, MPA, and right and left pulmonary arteries is presented in Table 1. PDA evaluation revealed the presence of calcifications and thrombi in $6(19 \%)$ and 1 (3\%) cases, respectively. The minimal, mean, and maximal diameters of PDA were $4.7 \pm$ $\pm 1.9 \mathrm{~mm}, 7.0 \pm 3.2 \mathrm{~mm}$, and $9.4 \pm 5.0 \mathrm{~mm}$, respectively. Fourteen patients with age $<40$ years tended to have a lower minimal diameter of PDA than others $(4.4 \pm 1.6 \mathrm{~mm}$ vs. $4.9 \pm 2.2 \mathrm{~mm}, \mathrm{p}=0.1)$. PDA types $A, B, C, D$, and $E$, classified according to Krichenko et al. [11], were seen in 16 (50\%), $3(9 \%), 9$ (28\%), $2(6 \%)$, and 2 (6\%) patients, respectively (Table 2$)$. The Spearman correlation coefficient between MPA and PDA diameters demonstrated a good correlation for minimal $(r=0.70, p<0.001)$, mean $(r=0.62$, $p=001)$, and maximal $(r=0.60, p=0.0003)$ PDA diameters. The Spearman correlation coefficient 
between right pulmonary artery and PDA diameters demonstrated a fair correlation for minimal $(r=0.57, p=0.007)$, mean $(r=0.52, p=0.003)$, and maximal ( $r=0.49, p=0.004)$ PDA diameters. The Spearman correlation coefficient between left pulmonary artery (LPA) and PDA diameters demonstrated a fair correlation for minimal $(r=0.46, p=$ $=0.008)$ and mean ( $r=0.40, p=0.02)$ PDA diameters while there was a poor correlation for maximal ( $r=0.39, p=0.03$ ) PDA diameter. The Spearman correlation coefficient between aortic isthmus and PDA diameters demonstrated a fair correlation for minimal $(r=0.46, p=0.009)$ and mean $(r=0.41$, $p=0.08)$ PDA diameters while there was a poor correlation for maximal $(r=0.27, p=0.14)$ PDA diameter. All evaluated PDAs were seen in a typical location and had a typically connected aortic isthmus and MPA-LPA connection site. Subjects with the type A configuration of PDA tended to have a larger diameter at the aortic orifice $(10.2 \pm 5.2 \mathrm{~mm}$ vs. $6.4 \pm$ $\pm 4.9 \mathrm{~mm}, \mathrm{p}=0.09$ ) and larger maximal diameter $(10.3 \pm 5.3 \mathrm{~mm}$ vs. $7.1 \pm 4.7 \mathrm{~mm}, \mathrm{p}=0.14)$ when compared to subjects with the type $C$ configuration.

\section{DISCUSSION}

The most common form of PDA is the type A morphology of PDA and comprised half (50\%) of the evaluated population. This observation is consistent with a study by Kirchenko et al. [11] which documented the occurrence of PDA with a wide aortic and narrow pulmonary orifice (type $A$ ) in the majority (64.5\%) of patients with PDA. We found the type C (tubular with wide aortic and pulmonary orifices) morphology of PDA to be the second most frequent (28\%), while in the previously mentioned study, the type B morphology of PDA (very short length, wide aortic and pulmonary orifices) was the second most frequent, with an occurrence of $17 \%$. There are a few possible explanations for this discrepancy. Firstly, we evaluated adult patients (mean age $41.4 \pm$ \pm 17.4 years), while Krichenko et al. [11] evaluated a paediatric (mean age $6.2 \pm 9.1$ years) population. The distribution of PDA morphology may differ with age. Secondly, in our observation, the type B PDA tended to have a shorter length and higher value of minimal diameter than the type C PDA, which may result in a larger shunt [9], more severe clinical symptoms, and early PDA closure intervention in childhood. Thirdly, Krichenko et al. [11] used the narrowest site of PDA as the landmark while we performed 3-di- mensional CT-based evaluation. This led to a more detailed morphometric qualitative and quantitative analysis, not only based on the measurement at the site of maximal narrowing, but also sites of aortic and pulmonary orifices and PDA length. In our quantitative analysis, subjects with the type A configuration of PDA compared to subjects with the type $C$ configuration tended to have a larger diameter at the aortic orifice and larger maximal diameter. Quantitative evaluation confirmed previous qualitative findings of larger aortic than pulmonary orifices in patients with the type A morphology of PDA (10.2 $\pm 5.2 \mathrm{~mm}$ vs. $5.1 \pm 2.7 \mathrm{~mm}, \mathrm{p}=0.0001)$. These observations are consistent with qualitative evaluation by Krichenko et al. [11]. In both studies, types D (with multiple constrictions) and $\mathrm{E}$ (elongated with distal constriction) ducts were marginal and made up only a few per cent of patients. Our qualitative CT analysis enabled detection of PDA thrombi and calcifications, which is essential in the planning of PDA percutaneous closure [12]. Comparison with previous studies regarding prevalence of PDA thrombus and calcification is limited due to the use of invasive angiography and a lack of specific data $[1,10,14]$.

Interestingly, in our study the mean value of narrowest PDA diameter was higher than in the above cited study $(4.8 \pm 1.9 \mathrm{~mm}$ vs. $3.2 \pm 1.0 \mathrm{~mm}$, $\mathrm{p}<0.0001)$. This could be due to a more accurate 2-dimensional measurement of the site (of maximal narrowing) using $\mathrm{CT}$ than the 1-dimensional measurement from invasive catheter angiography. Another reason for this discrepancy may be the different ages in the two evaluated populations. The minimal PDA diameter might have increased with age, and this theory supports our finding which is a tendency toward lower minimal PDA diameter in patients younger than 40 years of age when compared to others. Moreover, a minimal diameter $<3 \mathrm{~mm}$ favours intravascular occlusion [18], which might have occurred in childhood and excluded such patients from our population.

Patients with PDA had a larger diameter and dilatation of aortic isthmus and pulmonary arteries than individuals from the control group; however, no dilatation of the ascending and descending aorta was observed. Lack of ascending aortic dilatation is contrary to previous analyses $[5,10,11]$, while dilatation of aortic isthmus and pulmonary arteries is consistent with the above-mentioned publications. PDA diameters demonstrated the strongest correlation with MPA diameters and a decrease in its value was observed when com- 
pared with left and right pulmonary arteries, and aortic isthmus. This may result from the fact that all PDAs had a typical location and a typically connected aortic isthmus and site of MPA-LPA junction. Interestingly, aortic isthmus and pulmonary artery diameters had the strongest correlation with minimal PDA diameters and weakest correlation with maximal PDA diameters. This suggests that the degree of shunting through a PDA is limited by its narrowest, not its widest site, and that its significance influences dilatation of aortic isthmus and pulmonary arteries. To the best of our knowledge, this is the first such observation, which was enabled by quantitative $\mathrm{CT}$ evaluation.

The presence of clinical symptoms in patients with PDA is related to the magnitude of the shunt through the duct [14]. It was observed that patients with short and wide PDAs are more symptomatic than others [10]. Study by Evans et al. [6] classified ducts with diameter $>1.5 \mathrm{~mm}$ as haemodynamically significant, but it cannot be compared to our analysis as it was performed basing on echocardiographic evaluation in preterm infants. In our study performed in an adult population the mean value of the minimal diameter was more than 3 times greater and revealed $4.7 \pm$ $\pm 1.9 \mathrm{~mm}$. Other quantitative echocardiographic markers suggesting haemodynamically significant shunt in preterm infants are diastolic flow velocity in the LPA $>0.2 \mathrm{~m} / \mathrm{s}$ [17], increased left ventricular output [19] and increased peripheral vascular resistance index [15]. However, all above listed studies were different from our analysis which is morphological $C T$ based evaluation of PDA in an adult population. In premature infants significant shunt through PDA results in pulmonary hyperperfusion and peripheral hypoperfusion, which cause potentially fatal complications such as intraventricular haemorrhage, necrotising enterocolitis, kidney injury, heart failure, and chronic lung disease [4]. In an adult population clinical presentation of PDA may be completely asymptomatic in a small duct [7], while in a moderate to large duct the symptoms include tachycardia, exertional dyspnoea and hoarseness in presence of a very large duct $[7,14]$. Those symptoms occur due to development of congestive heart failure with pulmonary overcirculation and left heart volume overload. In clinical examination moderate to large ducts commonly reveal continuous heart murmur with concomitant hyperactive precordium and bounding peripheral pulses, while small ducts usually remain silent without presence of murmur [7]. To our best knowledge no evaluation of duct location and size measurements related to occurrence of clinical symptoms in an adult population was so far performed and is an interesting perspective missing in the existing literature. That is why lack of collection of clinical symptoms, diagnostics with evaluation of the magnitude of a shunt and follow up in the evaluated population is a major limitation of our study.

Unfortunately, the small patient population included in our study limits the statistical significance of the entire analysis. The study by Krichenko et al. [11] involved a larger patient population; however, they did not evaluate vessels other than PDA, which was done in our study. Finally, studies of PDA can be performed without ionizing radiation using magnetic resonance or echocardiography [16].

\section{CONCLUSIONS}

Computed tomography enables quantitative and qualitative evaluation of PDA, including its type of morphology, length, and diameter. In the evaluated adult population with PDA, the majority of patients had dilatation of aortic isthmus and pulmonary arteries. PDA diameters correlated with pulmonary artery diameters and this correlation was strongest between PDA diameter at the narrowest site and main pulmonary artery.

\section{REFERENCES}

1. Backer $\mathrm{CL}$, Mavroudis $\mathrm{C}$. Congenital heart surgery nomenclature and database project: patent ductus arteriosus, coarctation of the aorta, interrupted aortic arch. Ann Thorac Surg. 2000; 69(4 Suppl): S298-S307, doi: 10.1016/ s0003-4975(99)01280-1, indexed in Pubmed: 10798436.

2. Beerbaum $P$, Körperich $H$, Barth $P$, et al. Noninvasive quantification of left-to-right shunt in pediatric patients: phase-contrast cine magnetic resonance imaging compared with invasive oximetry. Circulation. 2001; 103(20): 2476-2482, doi: 10.1161/01.cir.103.20.2476, indexed in Pubmed: 11369688.

3. Carlgren LE. The incidence of congenital heart disease in children born in Gothenburg 1941-1950. Br Heart J. 1959; 21(1): 40-50, doi: 10.1136/hrt.21.1.40, indexed in Pubmed: 13618461.

4. El Hajjar M, Vaksmann G, Rakza T, et al. Severity of the ductal shunt: a comparison of different markers. Arch Dis Child Fetal Neonatal Ed. 2005; 90(5): F419-F422, doi: 10.1136/ adc. 2003.027698, indexed in Pubmed: 16113155.

5. Espino-Vela J, Cardenas N, Cruz R. Patent ductus arteriosus. With special reference to patients with pulmonary hypertension. Circulation. 1968; 38(1 Suppl): 45-60, doi: 10.1161/01. cir.38.1s5.v-45, indexed in Pubmed: 5712383.

6. Evans N, lyer P. Assessment of ductus arteriosus shunt in preterm infants supported by mechanical ventilation: effect of interatrial shunting. J Pediatr. 1994; 125(5 Pt 1): 
778-785, doi: 10.1016/s0022-3476(94)70078-8, indexed in Pubmed: 7965434.

7. Forsey JT, Elmasry OA, Martin RP. Patent arterial duct. Orphanet J Rare Dis. 2009; 4: 17, doi: 10.1186/1750-11724-17, indexed in Pubmed: 19591690.

8. Goitein O, Fuhrman CR, Lacomis JM. Incidental finding on MDCT of patent ductus arteriosus: use of CT and MRI to assess clinical importance. Am J Roentgenol. 2005; 184(6): 1924-1931, doi: 10.2214/ajr.184.6.01841924, indexed in Pubmed: 15908555.

9. Kim JH, Baek JH. Thoracic endovascular repair technique for the treatment of patent ductus arteriosus in an elderly patient: A case report. Medicine (Baltimore). 2018; 97(49): e13558, doi: 10.1097/MD.0000000000013558, indexed in Pubmed: 30544472.

10. Kimura-Hayama ET, Meléndez G, Mendizábal AL, et al. Uncommon congenital and acquired aortic diseases: role of multidetector CT angiography. Radiographics. 2010; 30(1): 79-98, doi: 10.1148/rg.301095061, indexed in Pubmed: 20083587.

11. Krichenko A, Benson LN, Burrows $P$, et al. Angiographic classification of the isolated, persistently patent ductus arteriosus and implications for percutaneous catheter occlusion. Am J Cardiol. 1989; 63(12): 877-880, doi: 10.1016/00029149(89)90064-7, indexed in Pubmed: 2929450.

12. Mitchell SC, Korones SB, Berendes HW. Congenital heart disease in 56,109 births. Incidence and natural history. Circulation. 1971; 43(3): 323-332, doi: 10.1161/01. cir.43.3.323, indexed in Pubmed: 5102136.
13. Pelberg R, Mazur W. Vascular CT angiography manual. Springer-Verlag, London 2003.

14. Schneider D, Moore J. Patent ductus arteriosus. Circulation. 2006; 114(17): 1873-1882, doi: 10.1161/circulationaha.105.592063.

15. Shimada S, Kasai T, Konishi M, et al. Effects of patent ductus arteriosus on left ventricular output and organ blood flows in preterm infants with respiratory distress syndrome treated with surfactant. J Pediatr. 1994; 125(2): 270-277, doi: 10.1016/s0022-3476(94)70210-1, indexed in Pubmed: 8040777.

16. Stout KK, Daniels CJ, Aboulhosn JA, et al. 2018 AHA/ ACC Guideline for the Management of Adults With Congenital Heart Disease: Executive Summary: A Report of the American College of Cardiology/American Heart Association Task Force on Clinical Practice Guidelines. Circulation. 2019; 139(14): e637-e697, doi: 10.1161/ CIR.0000000000000602, indexed in Pubmed: 30586768.

17. Suzmura H, Nitta A, Tanaka O. Diastolic flow velocity of left pulmonary artery of patent ductus arteriosus in preterm infants. Pediatr Int. 2001; 43: 146-151.

18. Waller BF. Morphological aspects of valvular heart disease: Part I. Curr Probl Cardiol. 1984; 9(7): 1-66, indexed in Pubmed: 6488868.

19. Walther FJ, Kim DH, Ebrahimi M, et al. Pulsed Doppler measurement of left ventricular output as early predictor of symptomatic patent ductus arteriosus in very preterm infants. Biol Neonate. 1989; 56(3): 121-128, doi: 10.1159/000243112, indexed in Pubmed: 2804176. 